\title{
A Variational Expression for the Relative Entropy
}

\author{
Dénes Petz
}

Mathematical Institute of HAS, Reàltanoda u. 13-15, H-1364 Budapest, PF.127, Hungary

\begin{abstract}
We prove that for the relative entropy of faithful normal states $\varphi$ and $\omega$ on the von Neumann algebra $M$ the formula

$$
S(\varphi, \omega)=\sup \left\{\omega(h)-\log \varphi^{h}(I): h=h^{*} \in M\right\}
$$
\end{abstract}

holds.

In general von Neumann algebras the relative entropy was defined and investigated by Araki $[1,3]$. After Lieb had proved the joint convexity of the relative entropy in the type $I$ case [10] several proofs appeared in the literature and they all benefited from the operator convexity of the function $t \rightarrow-\log t[8,11]$. Improving a result of Pusz and Woronowicz [14] Kosaki [9] obtained a variational formula for the relative entropy, which allows to extend the notion also to $C^{*}$-algebras. The expression we are going to deal with is of a different kind. It shows that the relative entropy $S(\varphi, \omega)$ as a function of $\varphi$ is the conjugate convex function (i.e., Legendre transform) of the convex function $h \rightarrow \log \varphi^{h}(I)$, where $\varphi^{h}$ denotes the inner perturbation of the state $\varphi$ by the selfadjoint operator $h$. The perturbed state $\varphi^{h}$ was used by Araki to extend the Golden-Thompson inequality $([7,18]$, see also [15]) to traceless von Neumann algebras. Approaching our variational expression for the relative entropy we generalize the GoldenThompson-Araki inequality [2] essentially and we state also the exact condition for the equality.

If $\varphi$ and $\omega$ are faithful normal states of the von Neumann algebra $M$ then the relative entropy is defined by means of the relative modular operator $\Delta(\varphi, \omega)$. If $\Omega$ is the vector representative of $\omega$ ) in the natural positive cone $P$ then

$$
S(\varphi, \omega)=-\langle\log \Delta(\varphi, \omega) \Omega, \Omega\rangle .
$$

The variational expression of Kosaki says that

$$
S(\varphi, \omega)=\operatorname{supsup}\left\{\log n-\int_{1 / n}^{\infty} t^{-1} \omega\left(y(t)^{*} y(t)\right)+t^{-2} \varphi\left(x(t) x(t)^{*}\right) d t\right\},
$$


where $y(t)=I-x(t)$, the first sup is taken over the positive integers and the second one is over all step functions $x:[1 / n, \infty) \rightarrow M$ such that the range of $x$ is finite and $x(t)=I$ for $t$ large enough.

For a cyclic and separating vector $\Phi \in P$ and a selfadjoint element $h \in M$ the perturbed vector $\Phi^{h}$ is defined by

$$
\Phi^{h}=\sum_{n=0}^{\infty} \int_{0}^{1 / 2} d t_{1} \int_{0}^{t_{1}} d t_{2} \ldots \int_{0}^{t_{n}-1} d t_{n} \Delta^{t_{n}} \Delta^{t_{n-1}-t_{n}} \ldots \Delta^{t_{1}-t_{2}} h \Phi,
$$

where $\Delta$ is the modular operator of $\varphi$. The perturbed functional is the nonnormalized vector functional corresponding to $\Phi^{h}$. The inequality

$$
\left\|\Phi^{h}\right\|^{2} \geqq \exp \varphi(h)
$$

reduces to the Golden-Thompson inequality if the algebra admits a faithful normal trace.

If $\varphi$ and $\omega$ are faithful normal states on the von Neumann algebra $M$ then $\omega$ is of the form $\varphi^{h}$ for some $h=h^{*} \in M$ provided that there are some constants $\lambda, \mu>0$ such that $\varphi \leqq \lambda \omega \leqq \mu \varphi[1]$. This $h$ is called the relative Hamiltonian.

Proposition 1. Let $\varphi$ and $\omega$ be faithful normal states on the von Neumann algebra $M$ and $h=h^{*} \in M$. Then

$$
\log \varphi^{h}(I) \geqq \omega(h)-S(\varphi, \omega),
$$

and the equality holds if and only if $\omega=\varphi^{h} / \varphi^{h}(I)$.

Proof. By Theorem 3.10 of [3] we have $S\left(\varphi^{h}, \omega\right)=S(\varphi, \omega)-\omega(h)$. The monotonicity of the relative entropy gives that $S\left(\varphi^{h}, \omega\right) \geqq \omega(I)\left[\log \omega(I)-\log \varphi^{h}(I)\right]$. Theorem 4 of [12] tells us that here the equality holds if and only if

$$
\left[D \varphi^{h}, D \omega\right]_{t}=\left(\varphi^{h}(I) / \omega(I)\right)^{i t} \quad(t \in \mathbb{R}),
$$

that is, $\varphi^{h}=\lambda \omega$ with a $\lambda \in \mathbb{R}^{+}$such that $\varphi^{h}(I)=\lambda \omega(I)$.

Corollary 2. $\log \varphi^{h}(I)=\sup \{\omega(h)-S(\varphi, \omega)$ : $\omega$ is a faithful normal state $\}$.

Corollary 3 (cf. [2]). The function $h \rightarrow \log \varphi^{h}(I)$ is convex on $M^{\text {sa }}$.

Theorem 4. Let $\alpha: M_{0} \rightarrow M$ be a unital 2-positive mapping between the von Neumann algebras $M_{0}$ and $M$, and let $\varphi$ be a faithful normal state of $M$. Assume that $\varphi \circ \alpha$ is a faithful normal state of $M_{0}$. Then for every $h=h^{*} \in M_{0}$, the inequality

$$
\varphi^{\alpha(h)}(I) \leqq(\varphi \circ \alpha)^{h}(I)
$$

holds. Furthermore, the equality implies $\varphi^{\alpha(h)} \circ \alpha=(\varphi \circ \alpha)^{h}$.

Proof. Let $\omega=\varphi^{\alpha(h)} / \varphi^{\alpha(h)}(I)$. Then

$$
\log \varphi^{\alpha(h)}(I)=\omega(\alpha(h))-S(\varphi, \omega)
$$

by Theorem 3.10 of [2] again. According to the monotonicity of the relative entropy $[9,11,16]$ we have

$$
S(\varphi, \omega) \geqq S(\varphi \circ \alpha, \omega \circ \alpha),
$$


and application of Proposition 1 gives that

$$
\log \varphi^{\alpha(h)}(I) \leqq(\omega \circ \alpha)(h)-S(\varphi \circ \alpha, \omega \circ \alpha) \leqq \log (\varphi \circ \alpha)^{h}(I) .
$$

If the latest inequality is actually an equality, then $\omega \circ \alpha=\lambda(\varphi \circ \alpha)^{h}$, that is $\varphi^{\alpha(h)} \circ \alpha$ $=\lambda(\varphi \circ \alpha)^{h}$.

Corollary 5. If $N \subset M$ and $h=h^{*} \in N$, then for a faithful normal state $\varphi$ on $M$ we have

$$
\varphi^{h}(I) \leqq(\varphi \mid N)^{h}(I)
$$

and the equality holds if and only if $\sigma^{\varphi}{ }_{t}(h) \in N$ for every $t \in \mathbb{R}$. In particular, if $N$ is commutative, then $\varphi^{h}(I) \leqq \varphi(\exp h)$ and $\sigma^{\varphi}{ }_{t}(h)=h$ for every $t \in \mathbb{R}$ is a necessary and sufficient condition for the equality.

Proof. We learn from the proof of the previous theorem that $\varphi^{h}(I)=(\varphi \mid N)^{h}(I)$ implies $S\left(\varphi^{h}, \varphi\right)=S\left(\varphi^{h}|N, \varphi| N\right)$, and due to Theorems 4 and 6 of [12] this is equivalent to the condition $\sigma^{\varphi}{ }_{t}(h) \in N$ for every $t \in \mathbb{R}$.

For a commutative $N$ we have $\psi^{h}(I)=\psi(\exp h)$ for every state $\psi$ on $N$ and

$\left\{a \in N: \sigma^{\varphi}{ }_{t}(a) \in N\right.$ for every $\left.t \in \mathbb{R}\right\}=\left\{a \in N: \sigma^{\varphi}{ }_{t}(a)=a\right.$ for every $\left.t \in \mathbb{R}\right\}$.

Corollary 5 is an extension of the Golden-Thompson-Araki inequality, which was proved in [2] by different methods. Our proof is based on the monotonicity of the relative entropy. Roughly speaking, the equality in Corollary 5 may occur only in a trivial way. It is so also in Theorem 4. The condition $\varphi^{\alpha(h)} \circ \alpha=(\varphi \circ \alpha)^{h}$ is very restrictive and its equivalent (formulated in terms of the modular groups) may be extracted from Theorems 2 and 8 of [13].

Theorem 6. Let $\left(p_{n}\right)$ be a sequence of projections in $M$ such that $p_{n} \rightarrow I$ strongly. If $M_{n}=p_{n} M p_{n}+\mathbb{C}\left(I-p_{n}\right)$, then

$$
S\left(\varphi\left|M_{n}, \omega\right| M_{n}\right) \rightarrow S(\varphi, \omega)
$$

as $n \rightarrow \infty$ for every faithful normal states $\varphi$ and $\omega$ on $M$.

Proof. Due to the monotonicity we have $S\left(\varphi\left|M_{n}, \omega\right| M_{n}\right) \leqq S(\varphi, \omega)$. Using Kosaki's formula we assume that

$$
\log n-\int_{1 / n}^{\infty} t^{-1} \omega\left(y(t)^{*} y(t)\right)+t^{-2} \varphi\left(x(t) x(t)^{*}\right) d t
$$

approximates $S(\varphi, \omega)$ for an appropriate step function $x:[1 / n, \infty) \rightarrow M$ with $x(t)=I$ for $t$ large enough. Set $x_{n}(t)=p_{n} x(t) p_{n}+\left(I-p_{n}\right)$ and $y_{n}(t)=I-x_{n}(t)$. Then

$$
S(\varphi, \omega) \geqq S\left(\varphi\left|M_{n}, \omega\right| M_{n}\right) \geqq \log n-\int_{1 / n}^{\infty} t^{-1} \omega\left(y_{n}(t)^{*} y_{n}(t)\right)+t^{-2} \varphi\left(x_{n}(t) x_{n}(t)^{*}\right) d t,
$$

and since

$$
\int_{1 / n}^{\infty} t^{-1} \omega\left(y_{n}(t)^{*} y_{n}(t)\right) t^{-2} \varphi\left(x_{n}(t) x_{n}(t)^{*}\right) d t \rightarrow \int_{1 / n}^{\infty} t^{-1} \omega\left(y(t)^{*} y(t)\right)+t^{-2} \varphi\left(x(t) x(t)^{*}\right) d t,
$$

we can conclude the theorem. 
Lemma 7. If $\varphi$ and $\omega$ are positive normal functionals on the von Neumann algebra $M$, then for every $n \in \mathbb{N}$ there is a projection $p \in M$ such that

$$
\varphi(\text { pap }) \leqq 2^{n} \omega(\text { pap }) \quad\left(a \in M_{+}\right)
$$

and

$$
\omega(I-p) \leqq 2^{-n} \varphi(I) .
$$

Proof. Let $\psi_{+}-\psi$ - be the Jordan decomposition of $\varphi-2^{n} \omega$ and let $p$ be $\operatorname{supp} \psi_{-}$ [17]. Then $\varphi($ pap $)-2^{n} \omega($ pap $)=-\psi_{-}($pap $) \leqq 0$ if $a \in M_{+}$. On the other hand, $\varphi(I-p)-2^{n} \omega(I-p)=\psi_{+}(I-p) \geqq 0$. So $\omega(I-p) \leqq 2^{-n} \varphi(I-p) \leqq 2^{-n} \varphi(I)$.

Proposition 8. If $\varphi$ and $\omega$ are faithful normal states on the von Neumann algebra $M$, then in any strong neighbourhood of the identity there is a projection q such that for some constants $\lambda, \mu \in \mathbb{R}^{+}$the estimate

$$
\varphi(q a q) \leqq \lambda \omega(q a q) \leqq \mu \varphi(q a q)
$$

holds for every $a \in M_{+}$.

Proof. We use the previous lemma twice. First, we choose a projection $p_{n}$ according to the lemma. Then we take the restrictions of $\varphi$ and $\omega$ to the subalgebra $p_{n} M p_{n}$ and change the roles. So we get a projection $q_{n} \leqq p_{n}$ such that

$$
\varphi\left(q_{n} a q_{n}\right) \leqq 2^{n} \omega\left(q_{n} a q_{n}\right), \quad \omega\left(q_{n} a q_{n}\right) \leqq 2^{n} \varphi\left(q_{n} a q_{n}\right) \quad(a \in M),
$$

and

$$
\varphi\left(p_{n}-q_{n}\right) \leqq 2^{-n} \omega\left(p_{n}-q_{n}\right) \leqq 2^{-n}, \quad \omega\left(I-p_{n}\right) \leqq 2^{-n}
$$

To show that $q_{n} \rightarrow I$ strongly it is sufficient to prove that $\varphi\left(I-q_{n}\right) \rightarrow 0$ (cf. $[6, \mathrm{I}$. Chap. 4, Proposition 4]). Indeed, $\omega\left(I-p_{n}\right) \rightarrow 0$ means that $p_{n} \rightarrow I$ strongly. Hence $\varphi\left(I-q_{n}\right)=\varphi\left(p_{n}-q_{n}\right)+\varphi\left(I-p_{n}\right) \rightarrow 0$.

Now we are in a position to prove the main result of the paper.

Theorem 9. If $\varphi$ and $\omega$ are faithful normal states on the von Neumann algebra $M$, then

$$
S(\varphi, \omega)=\sup \left\{\omega(h)-\log \varphi^{h}(I): h=h^{*} \in M\right\} .
$$

If the supremum is attained at $h=h^{*} \in M$, then $\omega=\varphi^{h} / \varphi^{h}(I)$.

Proof. We know both the inequality

$$
S(\varphi, \omega) \geqq \omega(h)-\log \varphi^{h}(I)
$$

and the condition for the equality from Proposition 1. A sequence $\left(p_{n}\right)$ of projections is guaranteed by Proposition 8 such that $p_{n} \rightarrow I$ strongly, and on the subalgebra $M_{n}=p_{n} M p_{n}+\mathbb{C}\left(I-p_{n}\right)$ the mutual majorization

$$
\varphi(a) \leqq \lambda_{n} \omega(a) \leqq \mu_{n} \varphi(a) \quad\left(0 \leqq a \in M_{n}\right)
$$

holds. Due to Theorem 6.3 of [1] the relative Hamiltonian for $\varphi_{n}=\varphi \mid M_{n}$ and $\omega_{n}=\omega \mid M_{n}$ exists. In other words, there is $h_{n} \in M_{n}, \omega_{n}=\left(\varphi_{n}\right)^{h_{n}}$. Hence

$$
S\left(\varphi_{n}, \omega_{n}\right)=\omega\left(h_{n}\right)-\log \left(\varphi_{n}\right)^{h_{n}}(I),
$$


and by Proposition 1 we have

$$
S\left(\varphi_{n}, \omega_{n}\right) \leqq \omega\left(h_{n}\right)-\log \varphi^{h_{n}}(I) .
$$

Since $S\left(\varphi_{n}, \omega_{n}\right) \rightarrow S(\varphi, \omega)$ in consequence of Theorem 6 we complete the proof by establishing $\omega\left(h_{n}\right)-\log \varphi^{h_{n}}(I) \rightarrow S(\varphi, \omega)$.

Acknowledgements. This paper was completed during the author's stay at the 2nd University of Rome in the framework of the "Year of Quantum Probability." The author is grateful to Professor Luigi Accardi for his kind hospitality.

\section{References}

1. Araki, H.: Relative Hamiltonian for faithful normal states of a von Neumann algebra. Publ. RIMS Kyoto Univ. 9, 165-209 (1973)

2. Araki, H.: Golden-Thompson and Peierls-Bogoliubov inequalities for a general von Neumann algebra. Commun. Math. Phys. 34, 167-178 (1973)

3. Araki, H.: Relative entropy of states of von Neumann algebras. Publ. RIMS Kyoto Univ. 11, 809-833 (1976)

4. Araki, H.: Relative entropy of states of von Neumann algebras. II. Publ. RIMS Kyoto Univ. 13, 173-192 (1977)

5. Bratteli, O., Robinson, D.W.: Operator algebras and quantum statistical mechanics. I, II. Berlin, Heidelberg, New York: Springer 1979 and 1981

6. Dixmier, J.: von Neumann algebras. Amsterdam: North-Holland 1981

7. Golden, S.: Lower bounds for Helmholtz functions. Phys. Rev. 137 B, 1127-1128 (1965)

8. Kosaki, H.: Interpolation theory and the Wigner-Yanase-Dyson-Lieb concavity. Commun. Math. Phys. 87, 315-329 (1982)

9. Kosaki, H.: Relative entropy of states: a variational expression. J. Operator Theory 16, 335-348 (1986)

10. Lieb, E.H.: Convex trace functions and the Wigner-Yanase-Dyson conjecture. Adv. Math. 11, 267-288 (1973)

11. Petz, D.: Quasi-entropies for states of a von Neumann algebra. Publ. RIMS Kyoto Univ. 21, 787-800 (1985)

12. Petz, D.: Sufficient subalgebras and the relative entropy of states of a von Neumann algebra. Commun. Math. Phys. 105, 123-131 (1986)

13. Petz, D.: Sufficiency of channels over von Neumann algebras. Quart. J. Math. Oxford (to appear)

14. Pusz, W., Woronowicz, S.: Form convex functions and WYDL and other inequalities. Lett. Math. Phys. 2, 505-512 (1978)

15. Simon, B.: Trace ideals and their applications. Cambridge: Cambridge University Press 1979

16. Uhlmann, A.: Relative entropy and the Wigner-Yanase-Dyson-Lieb concavity in an interpolation theory. Commun. Math. Phys. 54, $21-32$ (1977)

17. Takesaki, M.: Operator algebras I. Berlin, Heidelberg, New York: Springer 1980

18. Thompson, C.: Inequality with application in statistical mechanics. J. Math. Phys. 6, 1812-1813 (1965)

Communicated by $\mathrm{H}$. Araki

Received July 6, 1987 
